\title{
DYNAMO AND FOSSIL MAGNETIC FIELD IN YOUNG STARS
}

\author{
A.E. DUDOROV \\ Chelyabinsk State University, Russia
}

\begin{abstract}
The theory of fossil magnetic fields shows that new born stars may have internal magnetic fields of more than 1 million gauss. Convection inside young solar type stars will tangle any strong fossil magnetic field. The small scale magnetic field rises to the surface and determines the young stars activity attenuating with their age. When a fossil field is diminished a turbulent dynamo may begin to work in the condition of nonlinear stabilization. The scaling relations for the turbulent $\alpha \omega$ dynamo show that the strength of the generated "fossil" magnetic field inside the main sequence stars is stabilized on the level one tenth - 10 millions gauss, depending on the mass of the stars.
\end{abstract}

Key words: YOUNG STARS, CONVECTION, FOSSIL MAGNETIC FIELD, DYNAMO

\section{Observations of magnetic field}

The origin of the magnetic fields of stars is normally attributed to dynamo mechanisms operating in the regions of gyrotropic turbulence. The interstellar turbulence attenuates very fast in protostellar clouds and protostars. The battery mechanism is also very slow and inefficient in these objects.

There are now many observational data about the magnetic fields in the star forming regions (see Dudorov 1990; Troland 1990). An analysis of these observational data shows that there is a correlation between the magnetic field strength, $B$, and the numerical density $n$ of the star forming clouds:

$$
B \sim n^{k}
$$

with an exponent $1 / 2 \leq k \leq 2 / 3$. This relation and the data of optical and infrared polarimetry (see Meyers and Goodman 1991) show that the magnetic field accompanies the star formation on the density scale $n \approx 10^{0}-10^{10} \mathrm{~cm}^{3}$ or greater.

On the other hand we are sure now that the young stars such as T Tauri, Ae$B e$ stars have magnetic fields. Jonston and Penston $(1989,1990)$ concluded from statistical measurements of the Zeeman splitting that the star $R U$ Lup may have a magnetic field with $B \approx 500 \pm 260 G$. Saar (1991), Basry and Marcy (1991) estimated with Robinson technique that the magnetic field of T Tauri stars do not exceed the value $B \approx 1500 G$ and cover the $f_{s} \approx 0.1$ part of its surface. The radio observations, registration of XR flux from young stellar sources, disks, jets and stellar winds, magnetic activity of young stars and its attenuation with their age confirm the data of direct measurements. From this discussion we may conclude that magnetic fields of young stars may be a relic of the magnetic field of molecular and protostellar clouds.

\section{Theory of fossil magnetic field}

\subsection{OUTLINE OF THE THEORY}

The theory of fossil magnetic fields is based on numerical investigations of star formation in protostellar clouds with magnetic fields. The main goals of the theory 
are the study of the evolution of the magnetic flux in the processes of ambipolar and Ohmic diffusion, interaction with rotation, turbulence and MHD instabilities, that may develop on the various stages of protostellar clouds collapse, protostar and star contraction.

For these investigations we use the system MGD equations in the "diffusional" variables that consist of the usual MHD system and additional equations (see Dudorov, 1990):

$$
\begin{aligned}
& \frac{\partial x}{\partial t}+(\mathbf{v} \cdot \nabla) x=\frac{S_{p}}{\rho}-\frac{\nabla\left(x \rho \mathbf{v}_{m}\right)}{\rho} \\
& \frac{\partial \mathbf{v}_{m}}{\partial t}+(\mathbf{v} \cdot \nabla) \mathbf{v}_{m}=\frac{c_{s}^{2} \frac{\nabla x}{x}}{\rho_{i}}+\frac{\mathbf{F}_{e m}}{x \rho}-\eta_{i n} \rho \mathbf{v}_{m}, \\
& \frac{\partial \mathbf{B}}{\partial t}=\operatorname{curl}\left[\left(\mathbf{v}+\mathbf{v}_{m}\right) \times \mathbf{B}\right]-\nabla \times\left(\nu_{m} \nabla \times \mathbf{B}\right), \operatorname{div} \mathbf{B}=0
\end{aligned}
$$

where $x=\rho_{p} /\left(\rho_{p}+\rho_{n}\right), \mathbf{v}_{m}=\mathbf{v}-\mathbf{v}_{p}$ are the ionization degree and velocity of ambipolar diffusion, $\rho_{p}$ and $\rho_{n}$ are densities of charge and neutral components, $\mathbf{v}_{p}$ is the velocity of charge component, $S_{p}$ is the source function for the charges. Other quantities are used in the usual astrophysical meaning.

The equations (2-4) allow us to investigate the nonstationary and nonequilibrium ionization and nonstationary magnetic ambipolar diffusion (MAD) for magnetic field frozen into the plasma.

We elaborate the one and half approximation for the numerical solution of the MHD system in the diffusional variables in the case of weak-magnetic approach (Dudorov and Sazonov 1981, 1987). The numerical simulations are carried out with the help of modified Lax-Vendroff scheme. In a course of simulations we examine the thermal and ionization history in detail. We investigate the nonstationary and nonequilibrium ionization by cosmic rays, $\mathrm{XR}$ and radioactive elements with the thermal recombinations and recombination on the grains. We consider the thermal ionization of slender elements, hydrogen and helium and grains evaporation. Therefore we can study very carefully the ambipolar and Ohmic diffusion, magnetic detachment and interaction of magnetic field with rotation.

\subsection{Applications}

The numerical calculations show that the magnetic field is frozen into the gas in a course of isothermal collapse and acquire with time a quasi radial geometry besides the core. If the ionization state is determined by cosmic ray and radioactive elements, MAD diminish the magnetic field in the opaque protostellar core, when the central density $n_{c} \in\left[10^{5} n_{0}, 10^{9} n_{0}\right]$, where $n_{0}=10^{4}-10^{5} \mathrm{~cm}^{-3}$ is the initial density of the protostellar clouds. The adiabatic heating of the opaque core switches on the thermal evaporation of grains and thermal ionization of trace elements $\mathrm{K}, \mathrm{Na}, \mathrm{Al}$ e.c. with lower ionization potentials (Dudorov 1977a). Therefore in the regions with temperature $T \geq 4000-5000 \mathrm{~K}$ the magnetic field will be immersed into the whole gas again. The zone of powerful MAD moves to the surface in a course of the protostars and young stars evolution, coinciding with the region of minimal ionization 
degree. The attenuation of the magnetic flux relatively frozen in field was equal $\approx 10^{-2}$ for the Sun on the stars birth line.

The theory of fossil magnetic field has now a large number of applications (see Dudorov 1990). In the frame of this theory we have studied the magnetic braking during star formation; formation of protostellar magnetospheres; evolution of internal magnetic field of normal hot main sequence stars; ionizational and diffusional pumping of the magnetic field of chemically peculiar stars; escape of fossil magnetic field in the cool young star as the consequence of convective destruction of regular field, formation of magnetic flux tubes and their Ohmic decay and rising to the surface; dynamics of magnetic flux tubes and attenuation of magnetic activity of young stars.

\subsection{Main Conclusions}

Analytical estimations of the magnetic flux loss depending from screening of ionization and switch on of grain recombination show for the range of cosmic rays $r_{C R} \approx 130 \mathrm{~g} \cdot \mathrm{cm}^{-2}$, that the surface field,

$$
B_{s} \approx 200\left(\frac{\tau_{\kappa}^{2}}{T_{0}^{2}}\right) \cdot\left(\frac{M}{M_{\odot}}\right),
$$

where $T_{0} \approx 10 \mathrm{~K}$ and $M_{0}$ are the initial temperature and mass of the protostellar cloud, $\tau_{\kappa} \approx 10 \mathrm{~g} \cdot \mathrm{cm}^{-2}$ is the infrared optical depth (Dudorov and Tutukov 1990).

Numerical calculations for the large sample of various factors, changing the condition of star formation, allow us to obtain for the "deffreezing" factor $\beta_{f}=$ $B_{f} / B$, where $B_{f}$ and $B$ are the strength of frozen in and calculated magnetic field the following approximate formula:

$$
\beta_{f} \approx 10^{-2}\left(\frac{M}{M_{\odot}}\right)^{0.4-0.5}, M \approx 0.1-75 M_{\odot} .
$$

The surface magnetic field (before the interaction with convection),

$$
B_{s} \approx \tau_{C R}^{-2} \cdot Z_{R E} \cdot(a / q) \cdot \beta_{f}^{-1},
$$

depends on the "optical" depth for cosmic rays $\tau_{C R}$, the abundance of radioactive elements $Z_{R E}$, radius and abundance of grains $a$ and $q$. For the contemporary values of this parameters

$$
B_{s} \approx B_{s \tilde{0}} \cdot\left(\frac{M}{M_{\odot}}\right)^{0.25-0.35}, B_{s \hat{\mathrm{v}}} \approx f\left(\tau_{C R}, Z_{R E}, Z_{g}, \beta_{\hat{j}}\right) .
$$

$B_{s 0} \approx 1-100 G$ for normal stars and $B_{s 0} \leq 2000-3000 G$ for the magnetic $C p$ stars. The strength of the magnetic field is increased towards the center of the stars and in the core it has values of about $(1-10) \cdot 10^{6} G$ depending on the stellar mass.

\section{Fossil magnetic field and convection}

The investigation of the convective instability of a plane parallel layer with a magnetic field using the Boussinesq approach for compressibility, and linear theory of 
normal perturbations (Dudorov 1977b) allow us to formulate the following "convective theorem": In young stars with masses $M \leq 1.5 M_{\odot}$ on the stage of gravitational contraction to the main sequence, dynamic convection may develop without dependence on the magnetic field intensity if the magnetic energy $E_{m}<E_{g}$, where $E_{g}$ is the modulus of gravitational energy, because of strong opacity of partial ionized hydrogen and helium and infinite power of gravitation source.

Convective motions are turbulent, because of convective velocity $v_{k} \geq v_{A}$, Alfven's velocity and Reynolds number $R e \gg R e_{c r}$. Turbulent convective motions in the rotating stars destroy the regular fossil magnetic field and generate a chaotic small-scale turbulent magnetic field, include the dynamo-mechanisms after decreasing of fossil magnetic field strength lower than the level of nonlinear dynamo stabilization. The small scale magnetic field is dissipated by buoyancy and Ohmic decay, causing the magnetic activity of young cool stars (Dudorov 1991).

\section{Dynamo in young stars}

\subsection{Dynamo AND STRong SEed Fossil MAgnetic FiELD}

Investigations of fossil magnetic field evolution during star formation show that activity of cool stars approaching the main sequence must be supported by the dynamo-mechanisms. The precise time of switching of the fossil activity and switching on the dynamo generated activity, $t \approx 0.3 t_{K H}$, depends on the mass of stars and on the condition of star formation. Near to the switching time the fossil magnetic field has the energy $E_{m} \approx 0.3 E_{\text {turb }}$, which approximately equals the level of nonlinear dynamo stabilization (see Zel'dovich and Ruzmaikin 1987).

The investigation of magnetic field evolution in the stars leads to a new problem: How can the turbulent dynamo mechanisms develop under conditions, when the strength of the initial seed fossil magnetic field is near to the level of nonlinear stabilization or more? Intuitively we can say that in such a situation the fossil magnetic field must be supported at the level of nonlinear stabilization. This problem needs serious investigations. Our conclusions are confirmed by the scaling estimations.

\subsection{SCALING}

We can estimate the strength of a dynamo magnetic field on the basis of the equality of electromagnetic and Coriolis forces (see Dudorov et. al. 1989)

$$
B_{\varphi} B_{p} \approx 8 \pi \rho \omega L_{B} v_{t}
$$

where $\omega$ is the angular velocity, $L_{B}$ is the magnetic scale height and $v_{t}$ is the turbulent velocity. The toroidal and poloidal components of the magnetic field are connected by the equality:

$$
B_{\varphi} \approx D^{1 / 2} B_{p}, D \approx \alpha G L_{R}^{3} / \nu_{t} .
$$

We estimate the angular velocity gradient $G=r \partial \omega / \partial r$ with the help of numerical calculations of rotating star formation taking into account the magnetic braking 
and ambipolar diffusion. The calculations show that T Tauri stars of solar mass may have the following dependence of the angular velocity on the radius:

$\omega \approx \omega_{0}\left(\frac{r}{R}\right)^{-0.6}, \omega_{0} \approx 1.44 \cdot 10^{-5}$,

Taking into account, that the value of alfa effect $\alpha \approx v_{t}$, turbulent viscosity $\nu_{t} \approx v_{t} H_{P} / 3$ and $L_{B}=\zeta_{B} H_{P}$ we get for dynamo number:

$D \approx 5.4 \omega \zeta_{B}^{3} H_{P} v_{t}^{-1}$

It is easy to get with the help of formulas 9-12 the following scaling estimations:

$$
\begin{aligned}
& B_{p} \approx 3.3 \rho^{1 / 2}\left(\omega \zeta_{B}^{-1} H_{P} v_{t}^{3}\right)^{1 / 4}, \\
& B_{\varphi} \approx 7.7\left(\rho^{2} \omega^{3} \zeta_{B}^{5} H_{P}^{3} v_{t}\right)^{1 / 4},
\end{aligned}
$$

Estimations of the strength of toroidal and poloidal dynamo magnetic fields are provided for the convective envelope model of a star with solar mass, situated near to stars birth line. The real significance has the dependence of the basic parameters on the radial coordinate. The distribution of the magnetic field inside a young star is similar to a power law on the radius with exponent $\approx 1.7$. The strength of the poloidal field near the center of the star is $B_{p} \approx 2 \cdot 10^{6} G$, the strength of the toroidal field is more than 7 times.

The estimations for a sample of main sequence stars show that the strength of the dynamo supported fossil magnetic field inside the young stars may have the values $\approx(0.1-10) \cdot 10^{6} G$. Such a strength of the toroidal magnetic field is quite enough for the formation of magnetic ropes and their rising to the surface for Alfvén time.

\section{References}

Basry, G. and Marcy, G.W.: 1991, Lect. Not. Phys. 380, 401-406

Dudurov, A.E.: 1977a, in I.G.Kolesnik, ed(s)., Early Stage of Stellar Evolution, In Russian, Kiev: Naukova Dumka, 56-65

Dudorov, A.E.: 1977b, Nauchnye Informatsii Astronomicheskogo Soveta AN SSSR, In Russian 37, $1-10$

Dudorov, A.E.: 1990, Itogy Nauki i techniky. Astronomia, in Russian 39, 76-158

Dudorov, A.E: 1991, Pisma v Astron. Zh. 17, 521-529

Dudorov, A.E. and Sazonov, Yu.V.: 1981, Nauchnye Informatsii Astronomicheskogo Soveta AN SSSR, In Russian 49, 114-134

Dudorov, A.E. and Sazonov, Yu.V.: 1987, ibid 63, 68-86

Dudorov, A.E. and Tutukov, A.V.: 1990, Sov. Astron. 34, 171-175

Dudorov, A.E., Krivodubskii, V.N., Ruzmaikina, T.V. and Ruzmaikin, A.A.: 1989, Sov. Astron. 33, 420-426

Jonston, R.M.: 1987, Mon. Not. Roy. Astron. Soc. 227, 797-800

Jonston, R.M. and Penston, M.W.: 1986, Mon. Not. Roy. Astron. Soc. 219, 927-941

Meyers, P.C. and Goodman, A.A.: 1991, Astrophys.J. 373, 509-524

Saar, S.H.: 1991, Lect. Not. Phys. 380, 391-405

Troland, Th.: 1990, in R.Beck et.al., ed(s)., IAU Symp. N 140. Galactic and Intergalactic Magnetic Field, Dordrecht: Kluver Ac. Publ., 293-300

Zel'dovich, Ya.B. and Ruzmaikin, A.A.: 1987, Sov. Phys. Usp. 30, 494-506 